\title{
Innovative vibration technique applied to polyurethane foam as a viable substitute for conventional fatigue testing
}

\author{
Alexander Peralta ${ }^{1}$, Frederick Just-Agosto ${ }^{1, *}$, \\ Basir Shafiq ${ }^{2}$ and David Serrano ${ }^{1}$ \\ ${ }^{1}$ Department of Mechanical Engineering, University of \\ Puerto Rico, Mayaguez, Puerto Rico 00681, \\ e-mail: frederick.just@upr.edu \\ ${ }^{2}$ Department of General Engineering, University of Puerto \\ Rico, Mayaguez, Puerto Rico 00681 \\ * Corresponding author
}

\begin{abstract}
Lifetime prediction using three-point bending (TPB) can at times be prohibitively time consuming and costly, whereas vibration testing at higher frequency may potentially save time and revenue. A vibration technique that obtains lifetimes that reasonably match those determined under flexural TPB fatigue is developed. The technique designs the specimen with a procedure based on shape optimization and finite element analysis. When the specimen is vibrated in resonance, a stress pattern that mimics the stress pattern observed under conventional TPB fatigue testing is obtained. The proposed approach was verified with polyurethane foam specimens, resulting in an average error of $4.5 \%$ when compared with TPB.
\end{abstract}

Keywords: fatigue; polyurethane foam; three-point bending; vibration.

\section{Introduction}

Fatigue lifetime assessment of engineering materials is a crucial step between the design and use of products. Newer and more robust composite materials are increasingly becoming popular in all aspects of engineering product development. However, their success in the marketplace is predicated upon their ability to ensure safety. As a result, enormous effort has been devoted to fatigue lifetime characterization of a wide variety of engineering materials and products, including sandwich composites and foam cores as evidenced by the abundant literature [1-14]. Fatigue lifetime studies address a wide array of issues such as the effects of frequency, notches, damage accumulation mechanisms, testing environment, and stress level, to name a few. However, most of the fatigue lifetime characterization is based on the low-frequency testing of the specimens due mainly to the limitations imposed by the equipment $[15,16]$.

Furthermore, a high probability of disagreement is seen in the fatigue literature, as the material system and testing conditions vary inconsistently from one laboratory to another $[2,3]$.

Fatigue life behavior, in general, is notoriously stochastic in nature, especially in heterogeneous materials, such as composite materials $[1,2,7,8]$. As a result, multiple repetitions are required to obtain meaningful statistical data for analysis [15]. It is easy to see that when a million cycles or more is required per specimen, testing may consume significant time and revenue at the normal operational frequencies of $1-5 \mathrm{~Hz}$ in conventional servo-hydraulic machines, as illustrated in Table 1. Alternatives, such as high-frequency (up to $100 \mathrm{~Hz}$ ) machines are available to expedite the fatigue testing time, however, at a significant initial cost of purchase. Issues such as effect of frequency (inertia) and temperature changes have mainly been linked to high-frequency testing [2, 12, 17-20].

Similarly, vibration techniques have long been used to assess damage and product lifetime [21-31]; however, the vibration-based lifetimes and conventional flexural fatigue lifetimes seldom match due primarily to a mismatch in the generated stress pattern, loading type, frequency of loading, and frequency-induced temperature changes. Interest in vibration testing has primarily been focused on damage detection based on modal properties, size effect, impact, and nondestructive evaluation [32-36], to name a few.

The current research effort aims to refine an effective vibration technique that can be used to determine the fatigue life of a variety of engineering materials. What sets this technique apart from the conventional vibration testing is that through shape optimization of the test specimen, a stress pattern mimicking the stress pattern obtained in flexural fatigue testing is obtained. The main thesis behind the approach is that as long as the stress patterns match, the resulting lives obtained from fatigue and vibration testing must also correspond. This should be valid as long as frequency effects do not affect material properties significantly. The main differences between the current approach and work performed previously [29-31] is that, for the first time, the technique is actually verified against low-frequency flexural fatigue tests; it is applied to foam core material found in sandwich composites as opposed to homogeneous metals. Furthermore, the vibration frequency is performed at two orders of magnitude lower than that previously used and yet an order of magnitude higher than that normally used in three-point bending (TPB) $[30,31]$. Additionally, the first flexural vibration mode is used in this case, as opposed to higher torsional modes used by Scott et al. [29] and George et al. [30, 31].

Much of conventional fatigue testing is performed between 1 and $15 \mathrm{~Hz}$ of frequency (with inertial effects limiting testing frequency to below $5 \mathrm{~Hz}$ for most engineering materials). 
Table 1 Testing cost analysis at various frequencies. Total life: $10^{6}$ cycles at $\$ 1 / \mathrm{h}$.

\begin{tabular}{lllll}
\hline $\begin{array}{l}\text { Frequency } \\
(\mathrm{Hz})\end{array}$ & $\begin{array}{l}\text { Days per } \\
\text { data point }\end{array}$ & \multicolumn{2}{l}{ Total cost } & \\
\cline { 3 - 5 } & & $\begin{array}{l}1 \text { data } \\
\text { point }\end{array}$ & $\begin{array}{l}5 \text { data } \\
\text { points }\end{array}$ & $\begin{array}{l}10 \text { data } \\
\text { points }\end{array}$ \\
\hline 1 & 11.57 & 277 & 1385 & 2770 \\
10 & 1.157 & 27.7 & 138.5 & 277 \\
\hline
\end{tabular}

Within this range, frequency has been shown to affect the resulting lifetime; however, contradictory opinions of whether an increase in frequency increases or decreases the lifetime are present in the literature [17-19]. Not surprisingly, the predominant argument accompanying the effect of frequency relates to temperature-induced degradation of the material at higher frequencies. The temperature rise is primarily based on deformation induced intralayer/constituent friction of the composite materials $[2,12]$. Needless to say, both frequency and temperature are strongly dependent upon the material system used and testing environment [17-19]. In foams tested under laboratory-controlled temperature conditions, the heat dissipation is more likely to overcome any significant temperature rise $[1,2]$. Current vibration and fatigue experiments on closed cell polyurethane foams suggest a maximum increase in temperature of about $2^{\circ} \mathrm{C}$, which is not expected to influence the fatigue damage characteristics of the material.

Failure in sandwich composites is normally observed in the form of core shear under static and fatigue loading $[1,8]$; therefore, it is quite relevant to study the foam by itself to gain a better understanding of its predictive behavior. Furthermore, foam cracking process is less erratic as compared with the inherently stochastic sandwich composites, thus providing better reliability in establishing the effectiveness of the proposed technique. Therefore, the proposed methodology is used to study the fatigue lifetime characteristics of polyurethane foam used in sandwich composites. Results showed reasonably good correlation data to justify using the shape-optimized vibration technique as a viable substitute for the conventional low-frequency fatigue testing. The greatest advantage of this technique, when applicable, is significant savings in terms of testing time and revenue when applicable.

\section{Analytical framework}

The vibration specimen was designed with geometric shape synthesis and finite element analysis (FEA) modeling using brick elements. As the process is iterative, modeling was verified by comparing the predicted FEA's first and second natural frequencies with that of the initial rectangular FR7140 foam [37] beam used in the design process. This yielded errors of $2.41 \%$ and $2.77 \%$, respectively, as shown in Table 2. Vibration analysis (see in Figure 1) showed that the first natural frequency yields significantly higher stress amplitudes as compared with the second natural frequency. Because the highest stress values occur at the cantilever end, significant
Table 2 Comparison of experimental and predicted natural frequencies.

\begin{tabular}{lccc}
\hline $\begin{array}{l}\text { Natural } \\
\text { frequency }\end{array}$ & Experimental & $\begin{array}{l}\text { Predicted } \\
\text { frequency }\end{array}$ & $\begin{array}{l}\text { Percent } \\
\text { error }\end{array}$ \\
\hline 1st & $19.5 \mathrm{~Hz}$ & $19.03 \mathrm{~Hz}$ & $2.41 \%$ \\
2nd & $106.5 \mathrm{~Hz}$ & $109.45 \mathrm{~Hz}$ & $2.77 \%$ \\
\hline
\end{tabular}

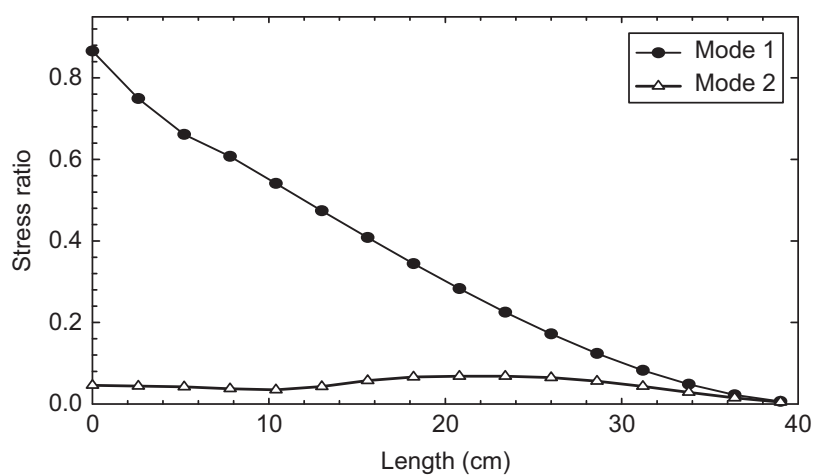

Figure 1 Comparison of the Von Misses centerline stress profile for the first two flexural transverse natural frequencies.

modification must be performed to mimic TPB in a given region of the vibration specimen. Changing either the loading pattern or the beam's geometry can readily accomplish this. The approach currently pursued is the latter of the two. To take advantage of the electromagnetic shaker characteristics (available force versus frequency), the starting point for the shape optimization was based on the stress amplitude pattern observed at first resonance.

The objective is to iteratively change the geometry of the cantilever vibration specimen such that a stress pattern similar to the TPB stress pattern is generated in a region of the cantilever while vibrating at its first resonance. As the electromagnetic shaker rating used for testing will be $<660 \mathrm{~N}$, a resonance limit of $40 \mathrm{~Hz}$ was implemented. The starting shape consists of a rectangular beam model with corresponding dimensions shown in Figure 2. During the optimization process, the width $(W)$ and the total length $\left(L_{3}\right)$ are kept constant to the original beams dimensions. The thickness at $H_{1}, H_{2}, H_{3}$, and $H_{4}$ corresponding to positions $0, L_{1}, L_{2}$, and $L_{3}$, respectively, are varied at $10 \%$ intervals. At each interval change, the natural frequency is calculated to verify that the first resonance does not exceed the frequency constraint. Modal analysis is also performed, and the stress pattern is determined to ensure that the changes are yielding the desired output. During each iteration step, amplitude and stress pattern

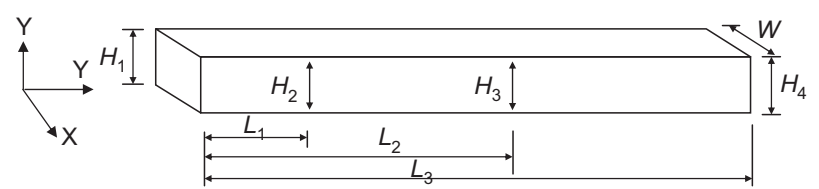

Figure 2 Parametric model used in geometric optimization. 

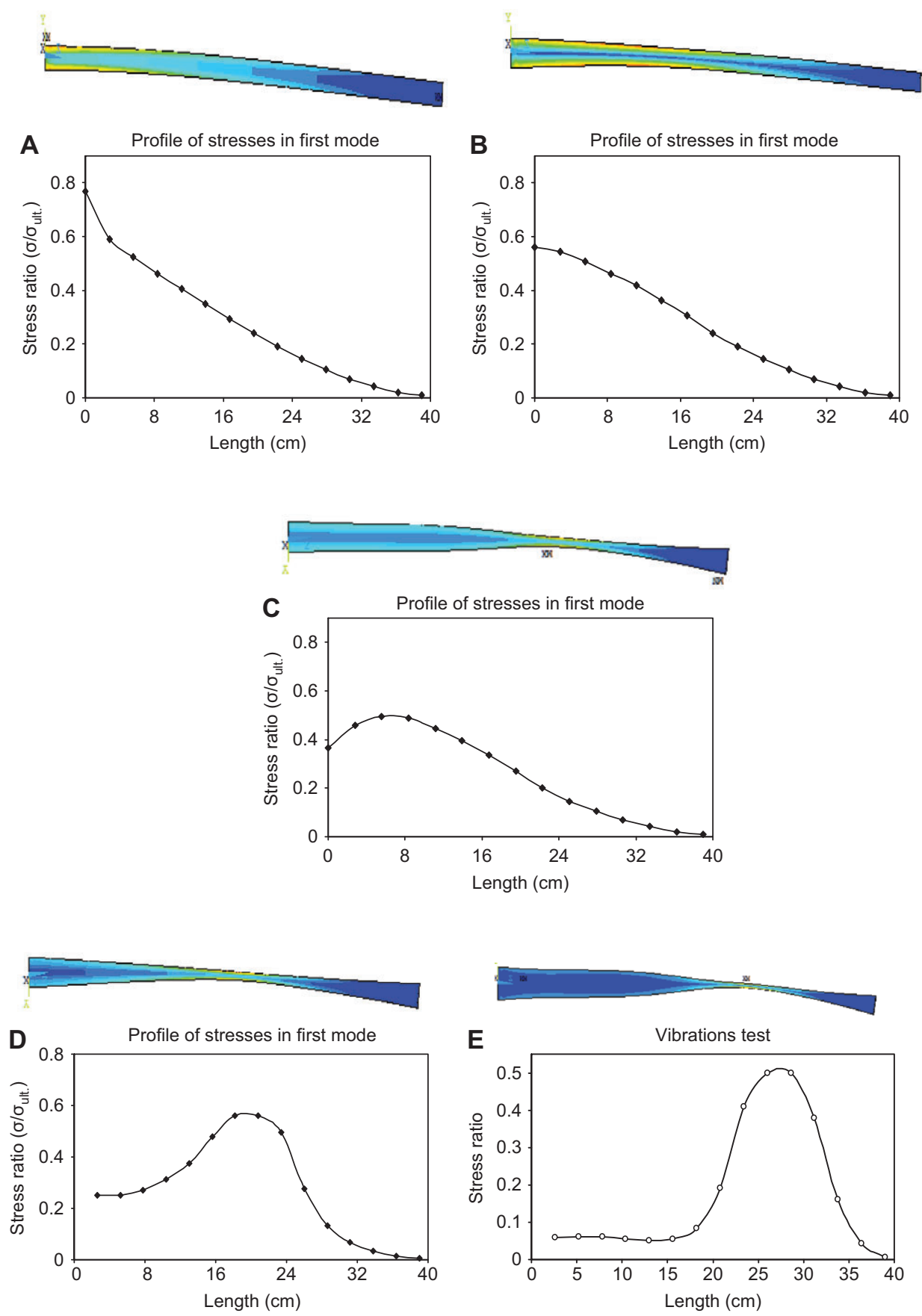

Figure 3 Geometric optimization process.

(A) Initial model, (B) increasing $H_{1}$, (C) decreasing $H_{2}$ and $H_{3}$, (D) increasing $H_{4}$, and (E) modifying $L_{1}$ and $L_{2}$.

changes occur. In addition, a shift of the stress pattern from the cantilever end is also observed. This behavior is shown in Figure 3A-E for various changes in $H_{1}, H_{2}, H_{3}, H_{4}$, and $L_{2}$ and $L_{3}$. When a good correlation in the region designed for fatigue (highest stress ratio) is obtained, the procedure is terminated. The final predicted stress pattern compared with TPB along with an image of the final test specimen is given in Figure 4. Figure 5 shows the final FEA cantilever beam model mesh along with an example of the double beam specimens that were manufactured for vibration fatigue analysis.

\section{Experimental setup}

TPB static and fully reversible $(R=-1)$ fatigue tests were performed on a servo-hydraulic MTS-810 machine 


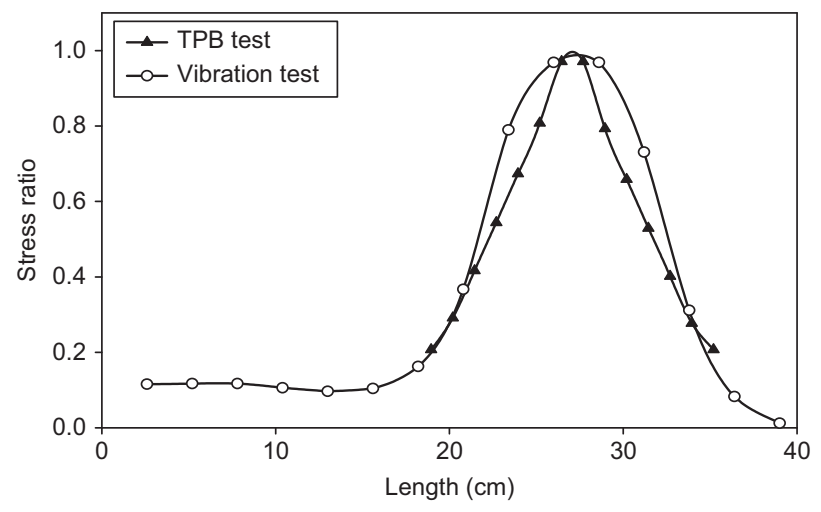

Figure 4 Comparison of the predicted Von Misses centerline stress profile from TPB specimens and vibration test specimens.

(MT8-80, MTS System Corporation, Eden Prairie, MN, USA). A 100-N/min loading rate was used for static testing, and a $0.75-\mathrm{Hz}$ sinusoidal waveform at stress ratios of 0.55 , $0.49,0.43$, and 0.39 was implemented for fatigue testing. To minimize the contact stresses and avoid indentation failure, butyl damping rubbers were used around the loading pin. A total of 16 specimens were tested using the setup shown in Figure 6.

Vibration testing included substantial effort in terms of specimen manufacturing based on the optimized shape. Specimen dimensions were determined using the FEA procedure, and dimensions were exported into CAD software to draw the geometry, which, in turn, was processed into the numerical control code for the CNC machining needed in generating the desired shape. Vibration testing was performed with an electrodynamic VTS-VG150 shaker, with a loading capacity of $150 \mathrm{lbf}$ and a maximum operating frequency of $4000 \mathrm{~Hz}$. Shaker input signals were generated using a SigLab dynamic signal analyzer. The experimental setup is depicted in Figure 7. Testing consisted of obtaining
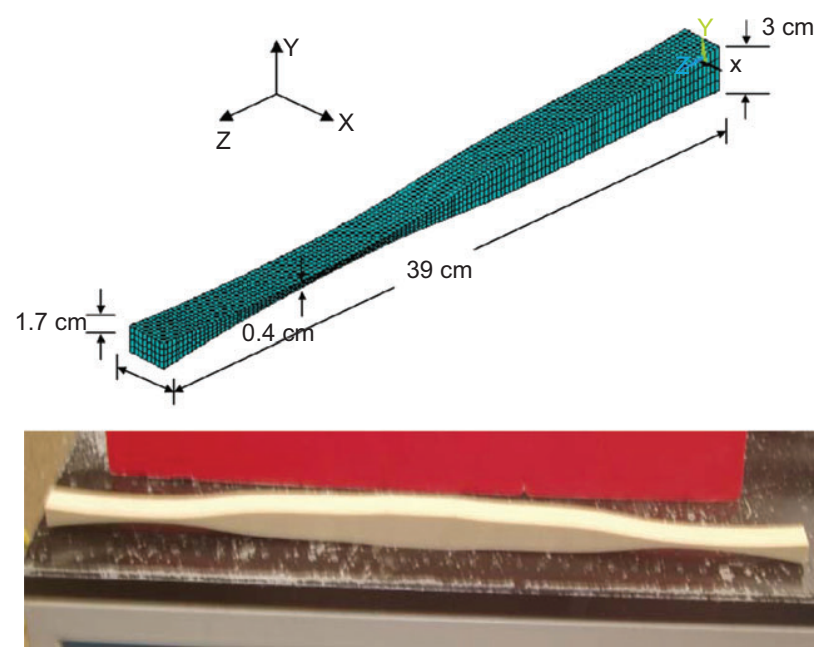

Figure 5 Optimized test FR7140 foam test specimen with meshing used for analysis.

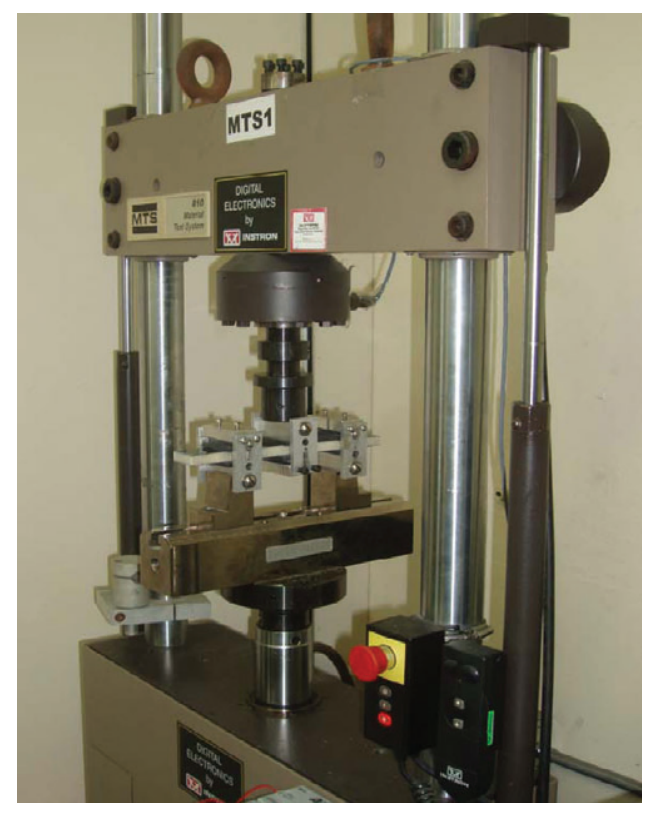

Figure 6 Testing setup used in TPB.

the natural frequencies from the frequency response functions (FRFs) and performing harmonic excitation at the first resonance frequency of $19.25 \mathrm{~Hz}$. The amplitude of the cyclic loading was determined using a force transducer attached to the holding jig located at the mid span of the double-cantilever configuration. Vertical displacements were recorded with a laser Doppler vibrometer (LDV), ensuring that the vibration specimen was vibrated with the predetermined resonance mode shape until failure occurred. Following this testing procedure, fatigue lifetime was obtained at stress ratios equivalent to those imposed under TPB tests, namely, $0.55,0.49,0.43$, and 0.39 . From the TPB and vibration fatigue testing data, S-N curves were generated.

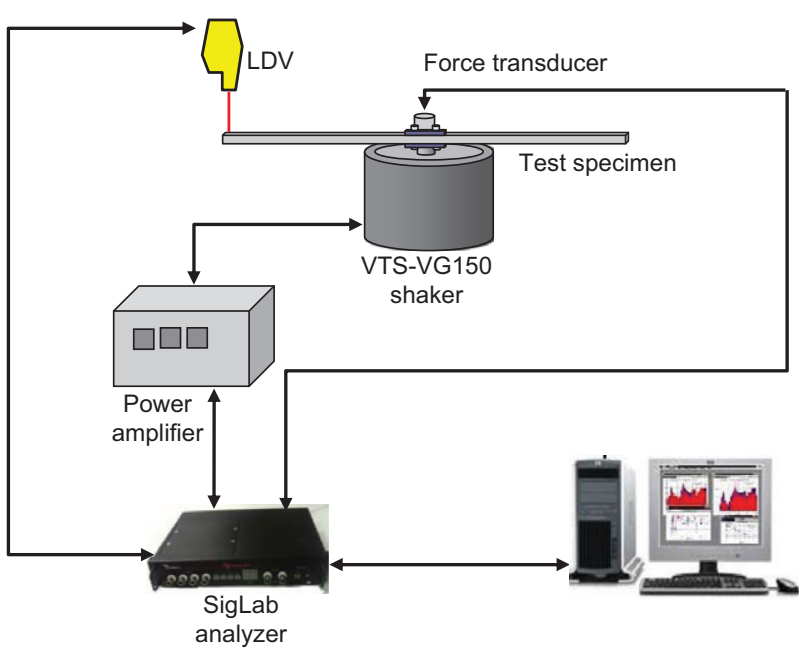

Figure 7 Experimental setup used for vibration fatigue testing. 


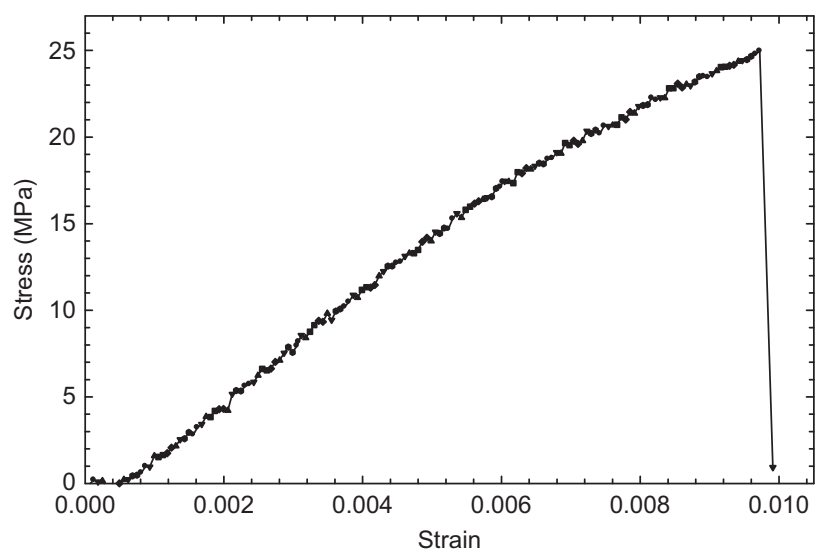

Figure 8 Experimental stress-strain diagram for specimen material using TPB.

\section{Results and discussion}

Using the ultimate static strength obtained from the stress strain curves (illustrated in Figure 8), fully reversible fatigue testing was performed at the aforementioned stress ratios. Results of the fatigue testing for both vibration testing and TPB are given in Figure 9. The observed increase in scatter at lower stress levels, as seen in both tests, is in agreement with similar behavior that has been reported in the literature [1]. By monitoring the input force and vertical displacement occurring during vibration testing, mode shapes yielded stress patterns that matched the stress levels obtained under TPB fatigue testing in the given vibration specimen design region. Initial results of TPB and vibration fatigue testing show a remarkable match in fatigue lifetime behavior as seen in Figures 9 and 10. Comparison of the fatigue and vibration test data clearly shows the two S-N lines to be almost parallel, indicating that the two techniques yield consistent results corresponding to the same material system. It is clearly evident that the fatigue life predicted from vibration testing is higher than that produced by TPB. One possible explanation for this behavior could be attributed to the effect of contact

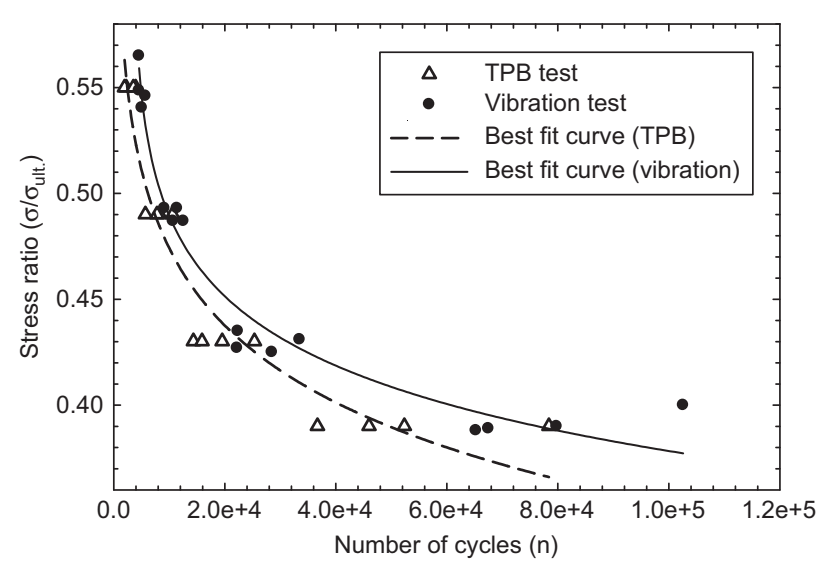

Figure 9 TPB and vibration S-N diagrams for FR7140 foam.

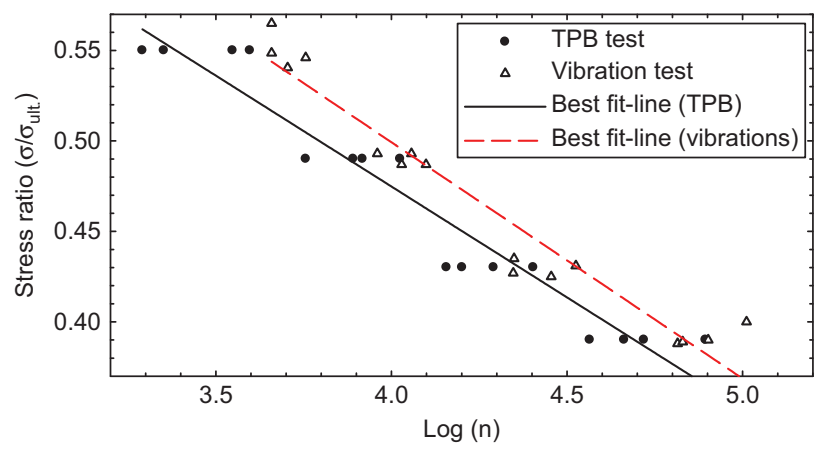

Figure 10 Semi-log plot of S-N TPB and vibrations S-N diagrams for FR7140 foam.

stress encountered in the jig used to hold the TPB specimens, whereas the vibration technique produces a contact-free moment, as shown in Figure 11. In Figures 9 and 10, the Von Misses centerline stress pattern values are calculated with a typical TPB test formulation [38] that does not consider the effect of contact stress. By considering the effect of contact stress [39, 40] [see Eq. (1)], the representative values of the actual Von Misses centerline stress occurring in TPB can be obtained.

$P_{\mathrm{o}}=\left(\frac{P^{\prime} E^{*}}{\pi R}\right)^{1 / 2}$

Here $P_{\mathrm{o}}$ is the stress caused by the maximum pressure over the specimen, $P^{\prime}$ is the applied force per unit length, $R$ is the effective curvature of the loading pin including the damping rubbers, and $E^{*}$ is the contact modulus that is determined by Eq. (2).

$\frac{1}{E^{*}}=\frac{1-v_{1}^{2}}{E_{1}}+\frac{1-v_{2}^{2}}{E_{2}}$

In Eq. (2), the modulus of elasticity and Poisson ratio for the damping rubber and foam are given by $E_{1}, v_{1}, \mathrm{E}_{2}$, and $v_{2}$, respectively. As a result of this new consideration, the Von Misses stresses at the surface centerline are now calculated with a compressive stress due to the contact stress and a tension as a result of the bending moment. Errors in predicting the material lifetime when compared with TPB predictions were reduced considerably yielding and average error of $4.5 \%$ with a maximum of $10 \%$ and a minimum of $-1 \%$ as shown in Figure 12. This error is most likely due to the inherent scatter in the data $[1,2]$. Thus, by adjusting the vibration data to represent the actual TPB stress occurring, a compatible prediction technique can be seen. Figure 13 shows the adjusted vibrations and TPB S-N diagram within the TPB $90 \%$ confidence levels. When compared with other vibration techniques that have been performed at high frequency, the results are very encouraging. Resonance testing with homogenous materials at frequencies $>1000 \mathrm{~Hz}$ obtained errors in the order of $20 \%$ or greater when compared with 

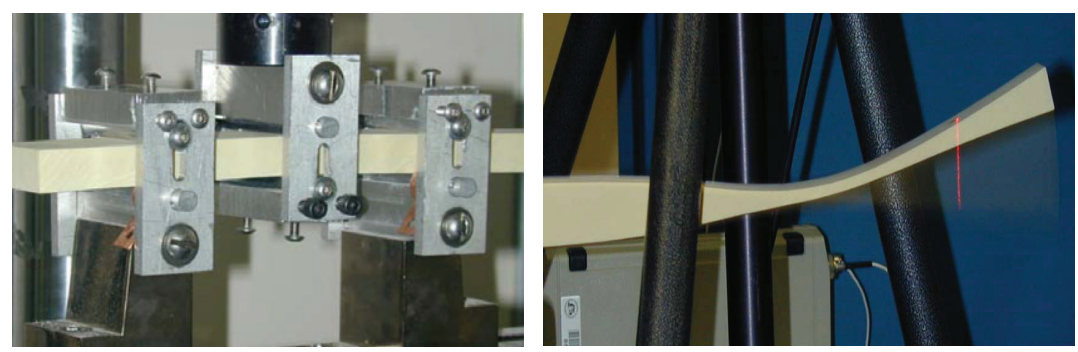

Figure 11 TPB testing demonstrating source of contact stress and vibration testing demonstrating free bending.

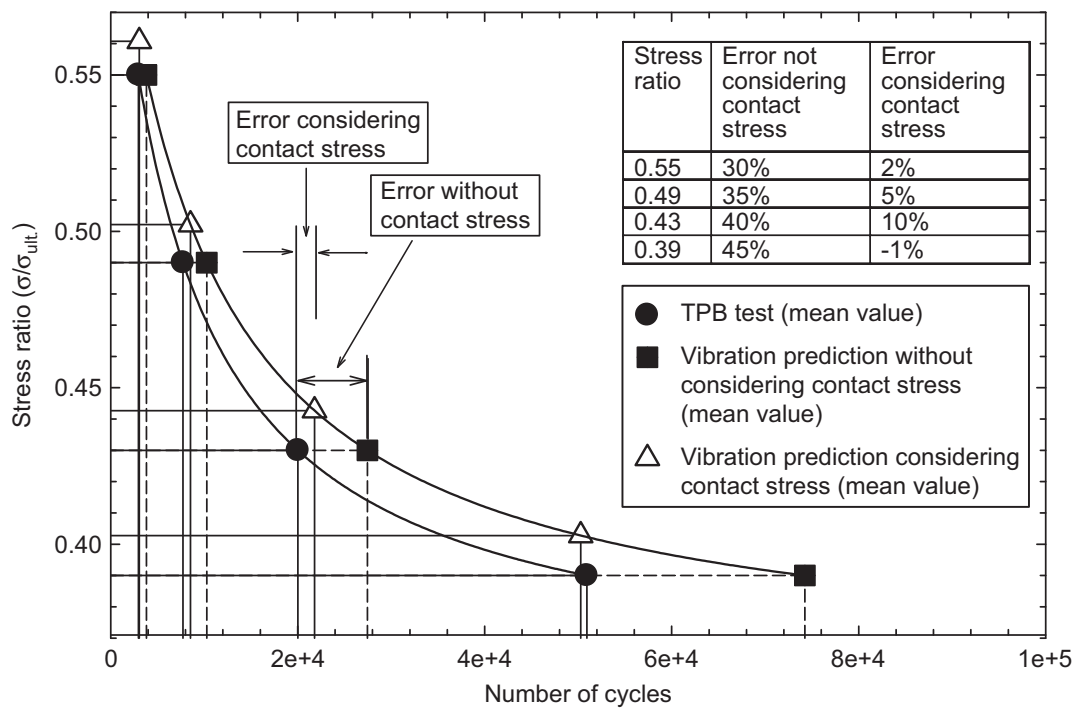

Figure 12 Effect of considering contact stress in lifetime evaluation.

conventional tension-tension fatigue lifetime predictions $[30,31]$. In addition, published literature investigating the effect of loading frequencies and temperature using a TPB configuration at 3 and $15 \mathrm{~Hz}$ obtained errors of $100 \%$ for fatigue life [12].

The increment of temperature under resonance was also examined before the specimen design process was ever

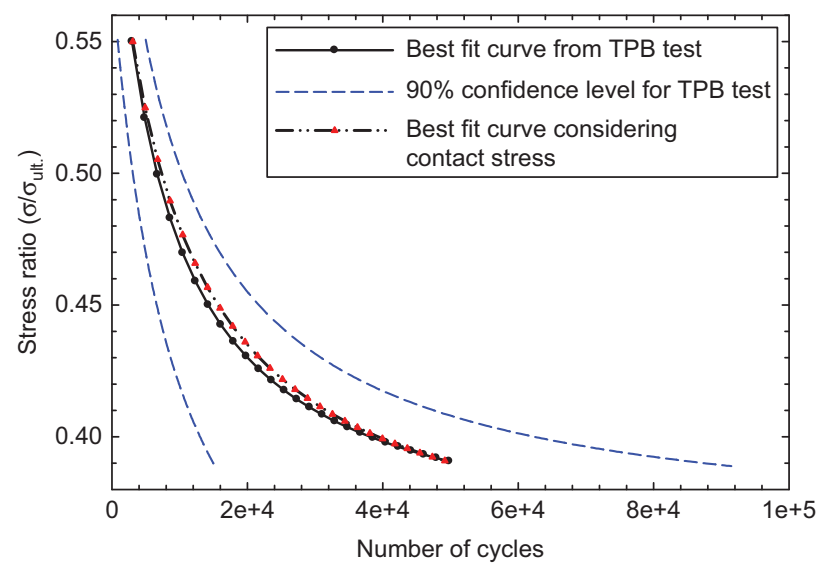

Figure 13 TPB and vibration S-N curve considering the effect of contact stress within the $90 \%$ confidence bounds for TPB. initiated. Here rectangular beams were vibrated at resonance for over 20-min periods, yielding a maximum temperature increase of $2{ }^{\circ} \mathrm{C}$ for all cases examined, which is considerably less than other works using TPB [12]. This is most likely due to the fact that the vibration technique does not have any direct contact, and while vibrating in resonance, a forced convention is present as compared with other commonly used methods in fatigue testing, TPB, for example.

In the flexural fatigue tests performed, it was very hard to find evidence of the process of fatigue crack nucleation and propagation, and there were no visual signs of damage in the specimens before an abrupt catastrophic failure. This type of behavior has been reported with fatigue testing of closed cell foams in the literature [1]. Due to catastrophic nature of crack propagation, $\mathrm{da} / \mathrm{dN}$ curves were not obtained.

Unlike homogeneous metals, the consistency of initial flaw size in foams required for crack propagation remains unclear. However, in closed cell foams of uniform density, it is reasonable to assume that the initial flaw size must be greater than the average cell diameter, perhaps resulting from inherently inconsistent cell wall properties of the foam. The larger average flaw size coupled with collapse and coalescence of a few neighboring cell walls can easily lead to an unsustained stress intensity required for ultimate crack propagation $[41,42]$. Once the crack nucleates, the ensuing crack growth is 
continuous and failure occurs abruptly in one cycle, similar to what has been observed in brittle homogeneous materials. In foams, the crack nucleates by failure and coalescence of individual cell links until a critical crack length leads to sufficient localized stress intensity needed to propagate the crack. Once this critical localized stress intensity is obtained, foam cells unable to cope with rising stresses collapse in rapid succession leading to abrupt failure observed. In foams, the cracks more often nucleate on the inside of the specimen thickness, making it difficult to detect the onset of crack initiation until it manifests on the surface, which, in many cases, is too late and has made some investigators to question whether it is of any real significance to know the initial flaw size $[1,2]$.

Under vibration testing, several observations were made. A change in the resonant frequency mode shape accompanied the onset of crack initiation before catastrophic failure, as local stiffness changes not observed on the beam's surface could be observed with the mode shape changes of the back-to-back cantilever setup. However, stiffness properties of foam being quite apart from the high-strength steel components did not yield changes in frequency during resonance vibration and could not be reliably associated with the extent of damage in the material. The forcing function had a significant bearing on the stress pattern. As there is no one-to-one correspondence between the forcing function and the stress magnitude, the forcing function magnitude has to be indirectly adjusted from the LDV displacements measurements and FEA. By tweaking the FEA results to match those produced by the forcing function, a reliable procedure was established to generate the desired stress magnitude and pattern. It should also be noted that materials having FRFs with low amplification factors (high damping) should not be considered because of the significant cost of the equipment needed to generate the high loads required. The goal of achieving a vibration methodology was satisfactorily achieved, but it should be cautioned that this procedure requires a precise specimen design as previously outlined to ensure proper results. The potential savings in revenue, especially when fatigue testing requires several data points such as that required for various stress levels to generate statistical S-N curves, further justifies the approach this time (25.667 faster).

\section{Conclusions and discussion}

The main aim of this investigation was to develop a vibration technique that, when applicable, can be used as an alternative method to obtain fatigue lifetime data with revenue savings. The main thesis behind the approach is that as long as the stress patterns match, those produced by TPB and that frequency effects are not significant, the resulting lives obtained from fatigue and vibration testing must also correspond. Sixteen specimens were submitted under TPB fatigue testing and 16 specimens were submitted under vibration fatigue testing, yielding an average error of $4.5 \%$ between their corresponding S-N curves. This alternative technique yielded S-N curve data at various stress levels 25.667 times faster than the convention TPB method. The mode of failure observed was catastrophic for both the TPB and vibrations methods, thus making it impractical to observe and monitor any crack growth. The method has shown to be an alternative that can have revenue savings especially when fatigue lifetime S-N curves require repetitive testing at given stress ratios.

\section{Acknowledgments}

This research was sponsored by ONR grant N00014-06-1-1043. The authors' thank Dr. Yapa S.S. Rajapakse, ONR Solid Mechanics program officer, for his mentoring, guidance, and support. Views expressed in this article are those of the authors and not of the program manager, funding agency, or their institutions.

\section{References}

[1] Zenkert D, Shipsha A, Burman M. J. Sandwich Struct. Mater. 2006, 8, 517-538.

[2] Sharma S, Gibson R, Ayorinde E. J. Sandwich Struct. Mater. 2006, 8, 263-319.

[3] Stone H. J. Cell. Plast. 1983, January/February, 47-60.

[4] Roosen D. J. Adv. Mater. 2006, 37, 38-42.

[5] Yau S, Mayer G. Mater. Sci. Eng. 1986, 78, 111-114.

[6] Noble F, Lilley J. J. Mater. Sci. 1981, 16, 1801-1808.

[7] Mahi A, Farooq M, Sahraoui S, Bezazi A. Mater. Des. 2004, 25 , 199-208.

[8] Kulkarni N, Mahfuz H, Jeelani S, Carlsson L. Compos. Struct. 2003, 59, 499-505.

[9] Caprino G. J. Compos. Mater. 2000, 34, 1334-1355.

[10] Clark SD, Shenoi RA, Allen HG. Compos. Sci. Technol. 1999, 59, 471-486.

[11] Berkowitz CK, Johnson WS. J. Compos. Mater. 2005, 39, 1417-1431.

[12] Kanny K, Mahfuz H, Thomas T, Jeelani S. Polym. Polym. Compos. 2004, 12, 551-559.

[13] Wu WF, Lee LJ, Choy ST. J. Compos. Mater. 1996, 30, 123-137.

[14] Epaarachchi JA, Clausen PD. Composites A. 2005, 36, 1236-1245.

[15] Collins JA. Failure of Materials in MechanicalAnalysis, Prediction, Prevention, 2nd ed. Hoboken, NJ: Wiley Interscience, 1993.

[16] Harris CM. Shock Vibration Handbook. Columbus, OH: McGraw-Hill, 1988.

[17] Kanny K, Mahfuz H. Compos. Struct. 2005, 67, 403-410.

[18] Sun C, Chan W. ASTM Spec. Tech. Publ. 1979, 674, 418-430.

[19] Barron V, Buggy M, McKenna N. J. Mater. Sci. 2001, 36, 1755-1761.

[20] Reifsnider KL, Stinchcomb WW, O'Brien TK. Fatigue of Filamentary Composite Materials, ASTM Spec. Tech. Publ. 636. Reifsnider KL, Lauraitis KN, Eds. ASTM International: Philadelphia, PA, 1977, pp. 171-184.

[21] Gibson RF. Compos. Sci. Technol. 2000, 60, 2769-2780.

[22] Liew KM, Xiang Y, Kitiponchai S. J. Sound Vibration 1995, 180, 163-176.

[23] Penn L, Jump J, Greenfield M, Blandford GE. J. Compos. Mater. 1999, 33, 54-72.

[24] Mead D, Markus S. J. Sound Vibration 1969, 10, 163-175.

[25] Kosmatka J, Ricles J. J. Struct. Eng. 1999, 125, 1384-1392.

[26] Salawu OS. Eng. Struct. 1997, 19, 718-723.

[27] Shu D. Compos. Sci. Technol. 1995, 54, 101-109.

[28] Peeters B, Maeck J, Roeck G. Smart Mater. Struct. 2001, 10, 518-527. 
[29] Scott O, Shen H, George T, Cross C, Calcaterra J. J. Eng. Gas Turbines Power 2007, 129, 162-169.

[30] George T, Seidt J, Shen M, Nicholas T, Cross C. Int. J. Fatigue 2004, 26, 477-486.

[31] George T, Shen M, Scott O, Nicholas T, Cross C, Calcaterra J. Trans. ASME 2005, 127, 58-64.

[32] Zou Y, Tong L, Steven G. J. Sound Vibration 2000, 230, 357-378.

[33] Ayorinde E. J. Compos. Mater. 1995, 28, 125-1039.

[34] Liew K, Xiang Y, Kitiponchai S. J. Sound Vibration 1995, 180, 163-176.

[35] Abrate S. Impact on Structures. Cambridge University Press: Cambridge, UK, 1998.

[36] American Society of Testing and Materials. Annual Book of the ASTM Standards. ASTM International: West Conshohocken, PA, 2007.
[37] General Plastics Manufacturing Company. FR7100 Foam Data Sheet. Tacoma, WA: General Plastics. www.GenralPlastics. com, 2005.

[38] American Society of Testing and Materials. ASTM D790-07e1, Standard Test Methods for Flexural Properties of Unreinforced and Reinforced Plastics and Electrical Insulating Materials. ASTM International: West Conshohocken, PA, 2007.

[39] Collins JA. Mechanical Design of Machine Elements and Machines, A Failure Prevention Perspective. John Wiley \& Sons: New York, NY, 2003.

[40] Bull J. Elastic Contact Stress Formulae. Viewed 2009. Available at: http://www.staff.ncl.ac.uk/s.j.bull/ecstr.html, 2006.

[41] Gibson LJ, Ashby MF. Cellular Solids - Structure and Properties, 2nd ed. Cambridge University Press: Cambridge, UK, 1997.

[42] Burman M, Zenkert D. Int. J. Fatigue 1997, 19, 551-561. 